\title{
THE EFFECTIVENESS OF TOPICAL DICLOFENAC IN RELIEVING DISCOMFORT FOLLOWING TRAUMATIC CORNEAL ABRASIONS
}

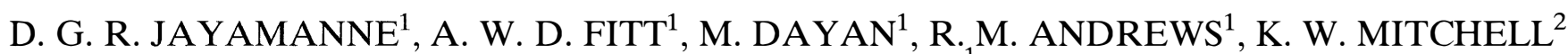 \\ and P. G. GRIFFITHS ${ }^{1}$ \\ Newcastle upon Tyne
}

\section{SUMMARY}

Diclofenac is a non-steroidal anti-inflammatory drug available in an ophthalmic preparation. We present a prospective randomised double-masked placebo-controlled trial involving 40 patients that assessed the effectiveness of topical diclofenac in relieving pain from traumatic corneal abrasions. Statistical analysis of visual analogue and categorical pain scores revealed a significant reduction in pain experienced by subjects in the diclofenac group $(p<0.02)$.

Traumatic corneal epithelial abrasion is a common reason for attendance at accident and emergency and eye departments. Patients with corneal epithelial injury experience significant ocular pain especially in the first $24-48$ hours, and often until corneal reepithelialisation. ${ }^{1}$ Despite the use of cycloplegics, patching and oral painkillers the pain is inadequately controlled in many patients. Topical anaesthetics are known to be toxic to the corneal epithelium and are therefore not normally used for analgesia. Corneal epithelial wounds heal by a process of cell migration and proliferation and the majority of these abrasions heal within 1-4 days. ${ }^{2,3}$

Diclofenac is a potent non-steroidal anti-inflammatory drug (NSAID) used to relieve the pain and inflammation in conditions such as rheumatoid arthritis, degenerative joint disease and other inflammatory conditions. ${ }^{4}$ An ophthalmic preparation of diclofenac sodium $0.1 \%$ solution is available for the treatment of post-operative inflammation.

We present the results of a prospective randomised double-masked placebo-controlled compara-

From: ${ }^{1}$ Department of Ophthalmology, Newcastle General Hospital, Newcastle upon Tyne; ${ }^{2}$ Regional Department of Medical Physics, Royal Victoria Infirmary, Newcastle upon Tyne, UK.

Correspondence to: Mr D. G. R. Jayamanne, FRCOphth, Department of Ophthalmology, Sunderland Eye Infirmary, Queen Alexandra Road, Sunderland SR2 9HP, UK. tive trial to determine the effectiveness of topical diclofenac in reducing pain caused by traumatic corneal abrasions.

\section{PATIENTS AND METHODS}

This study was a prospective double-masked comparison of diclofenac and placebo involving a total of 20 patients in each group and had the prior approval of the hospital ethics committee. Patients were recruited from the eye casualty department at Newcastle General Hospital. Those included in the study presented within 24 hours with a unilateral corneal abrasion and no other injury. Patients with previous corneal pathology, including dystrophies and recurrent erosion syndrome, diabetes, and those under 18 years of age or with known hypersensitivity to either NSAIDs or chloramphenicol were excluded. Written informed consent was obtained from all patients by the examining doctor.

Patients' pain was assessed in three ways (Fig. 1). Firstly, a visual analogue scale was used in which the patient placed an ' $x$ ' on a horizontal line measuring $10 \mathrm{~cm}$ in length showing a continuum from 'no pain' to 'worst pain ever'. Secondly, a categorical pain scale was completed, allowing the patient to describe the eye pain as none, mild discomfort not requiring painkillers, moderate pain requiring painkillers or severe disabling pain. Thirdly, pain was further subcategorised into foreign body sensation, light sensitivity and headache-like deep pain within the eye, and the patient was requested to describe the above categories as none, mild, moderate or severe.

Patients were randomly assigned to one of the two treatment groups: either diclofenac $0.1 \%$ or placebo (normal saline). The drops were dispensed in unmarked containers, to be used 4 times per day in the affected eye, in addition to the chloramphenicol ointment routinely used in this condition. Neither eye pads nor cycloplegics were used in order to

Eye (1997) 11, 79-83 ㄷ 1997 Royal College of Ophthalmologists 


\section{Visual analogue pain scale}

Please place an " $X$ " on the line below to indicate the amount of pain, if any, that you have felt over the last 24 hours.

\begin{tabular}{|c|c|c|c|}
\hline & Mil & Moderate & Severe \\
\hline
\end{tabular}

\section{Categorical pain scale}

The amount of pain in your affected eye over the last 24 hours:
1. None
2. Mild discomfort not requiring painkillers
3. Moderate pain requiring
4. Severe disabling pain painkillers

\section{Ocular descriptors}

Circle one number which describes the level of discomfort in each category that you have felt in your affected eye over the last 24 hours.

$\begin{array}{lcccc} & \text { None } & \text { Mild } & \text { Moderate } & \text { Severe } \\ \text { Foreign body sensation } & 0 & 1 & 2 & 3 \\ \text { Light sensitivity } & 0 & 1 & 2 & 3 \\ \text { Headache-like pain } & 0 & 1 & 2 & 3\end{array}$

Fig. 1. Patient questionnaire. Each patient completed these three assessments of their pain.

simplify the treatment regime, and patients were advised to take adequate oral analgesia as required. No specific instructions were given regarding the type or amount of oral analgesics to be taken, since the design of the categorical pain scale grouped all patients who took oral analgesics into the same category independent of the amount or type (Fig. 2).

Doctors involved in the patient assessments were masked as to the study drug codes. Provision was made for 'code breaking' in the event of adverse effects.

The patients were reviewed daily until complete corneal re-epithelialisation occurred, and at each visit a patient was asked to quantify his or her pain over the preceding 24 hours on the same scales. The size of the abrasion was also documented daily by measuring the greatest dimensions with the slit beam, in order to ensure that healing was occurring. A full slit lamp examination was also performed.

With regard to the statistical analysis of the data in the two groups, it was felt appropriate to use a distribution-free method, so the Wilcoxon rank sum test for two samples was employed. As differences between the two groups were expected in one direction only, single-tailed significance values are shown in the results. Data from day 0 to day 2 were compared.

\section{RESULTS}

All patients completed the study as planned and no unmasking of patients occurred during the trial. All patients in both groups had suffered relatively minor ocular trauma from accidental scratches with fingers, plants and stationery items. There were no adverse drug effects observed and all corneal abrasions were healed by 96 hours. No attempt was made to measure the area of the abrasions as this would have been impractical and inaccurate. Therefore, it was not possible to compare abrasion size with severity of pain.

The categorical pain scale data for day 0 (time of presentation), day 1 and day 2 are shown in Fig. 2 . On day 0 there was no statistically significant difference in the distribution of scores between the placebo and diclofenac groups. On day 1 the diclofenac score distribution was statistically smaller than that of the placebo (Wilcoxon rank sums $\left.T_{1}=338, T_{2}=482, N_{1}=N_{2}=20, p=0.025\right)$. By day 2 the difference had increased further but on reduced data sets (Wilcoxon rank sums $T_{1}=149.5, T_{2}=40.5$, $\left.N_{1}=9, N_{2}=10, p<0.001\right)$.

The visual analogue scale data presented substantially the same picture, albeit with higher significance on day 1 (Wilcoxon rank sums $T_{1}=355, T_{2}=506$, $\left.N_{1}=N_{2}=20, p<0.002\right)$.

Fig. 3a-c represents foreign body sensation, light sensitivity (photophobia) and headache-like pain during the first 48 hours. The placebo group experienced moderate or severe levels of discomfort in the three categories more frequently than did the diclofenac group. 

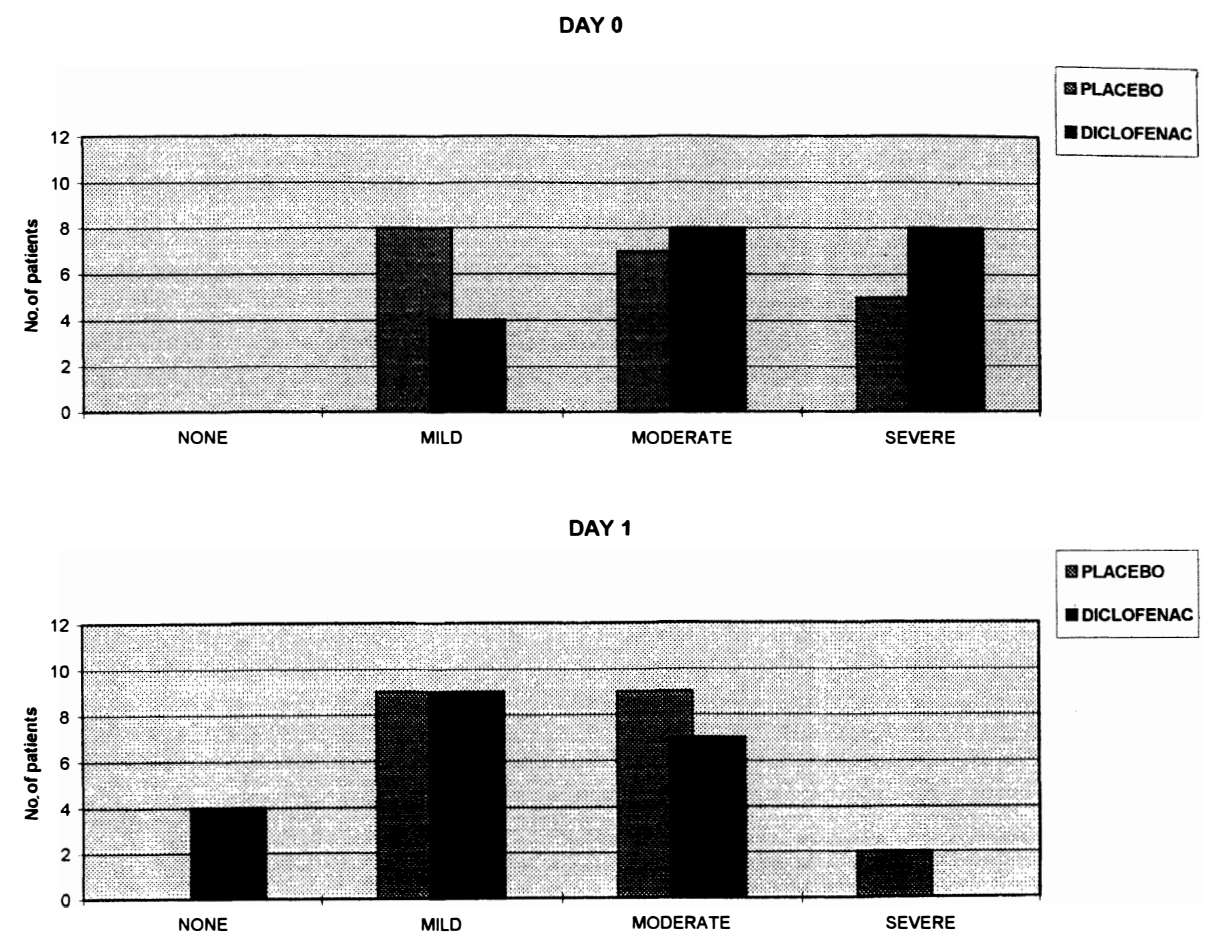

IDICLOFENAC

DAY 2

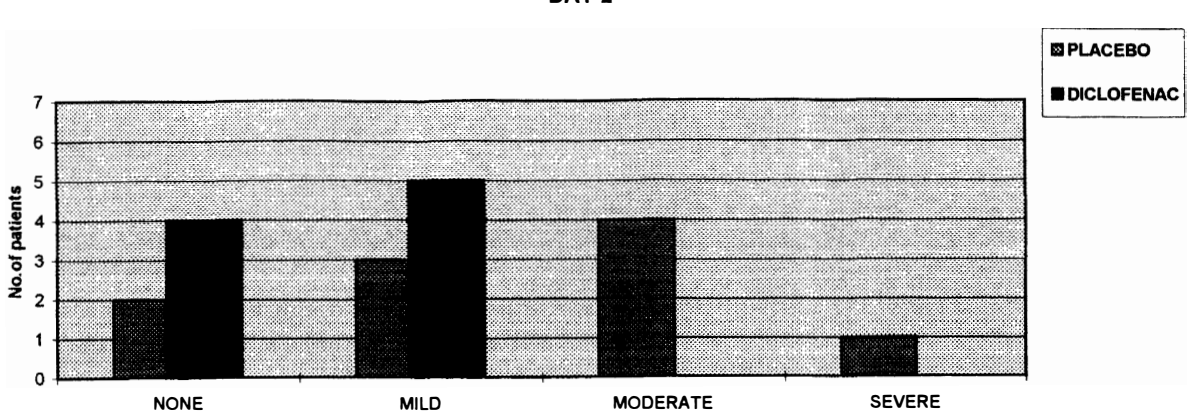

Fig. 2. Categorical pain scale data for day 0, day 1 and day 2 .

\section{DISCUSSION}

Traditional treatment of corneal abrasions includes antibiotic ointment, cycloplegics and oral analgesics. Eye pads are advocated by certain clinicians but at least one study has shown delayed healing with the use of pads and no improvement in patient comfort. ${ }^{5}$ Topical anaesthetic agents are avoided as these are believed to delay epithelial healing. The pain after corneal epithelial loss can be very severe and many patients are unable to return to work despite the above measures. ${ }^{1}$ A number of mechanisms may produce pain following traumatic corneal abrasions. Mechanical disruption of the epithelium can result in breakdown of cell membranes and the release of chemical factors such as prostaglandins, substance $\mathrm{P}$ and histamine. These chemical mediators have been shown to produce pain. ${ }^{6}$ Rapid re-epithelialisation after traumatic corneal abrasions is desirable to reduce risk of infection and eliminate pain. No evidence exists in the medical literature that diclofenac interferes with the rate of corneal epithelial healing. Indeed, diclofenac administered 4 times daily has been shown to have no effect on corneal wound healing or epithelial migration rate in animal models. ${ }^{7}$ There were no cases in our study of abrasions which failed to heal quickly in either group. A known cause of persistent epithelial defects is preservative toxicity. ${ }^{8}$ The preparation of diclofenac used in this study was preservative-free single dose units.

Recently, the pain following corneal abrasions following excimer laser photorefractive keratectomy has been successfully treated with diclofenac eye drops. ${ }^{1}$ Diclofenac is a potent non-steroidal antiinflammatory drug (NSAID). The mechanism of action of NSAIDs is the inhibition of the enzyme cyclo-oxygenase. ${ }^{4}$ Cyclo-oxygenase inhibitors produce some of their effect by inhibiting the production 

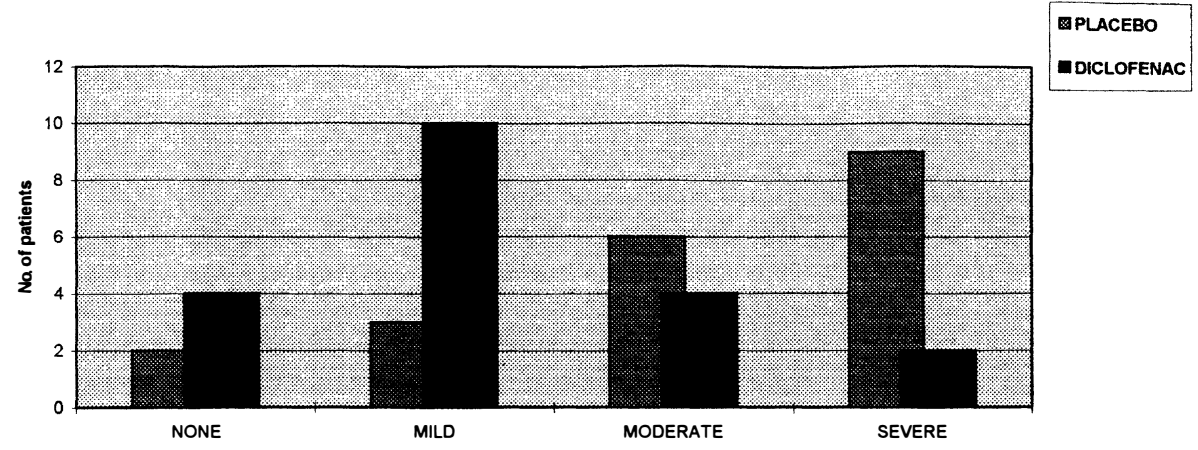

(a)

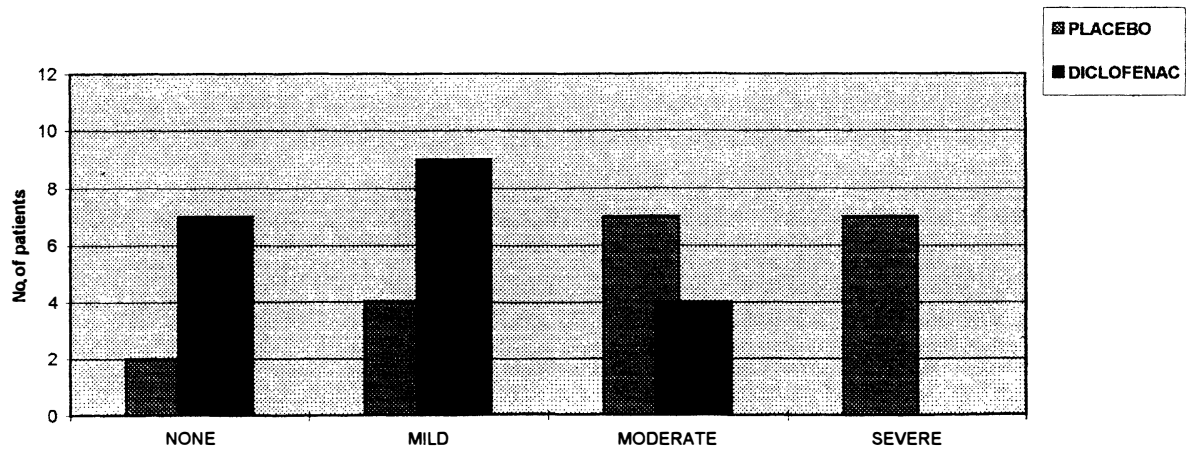

(b)

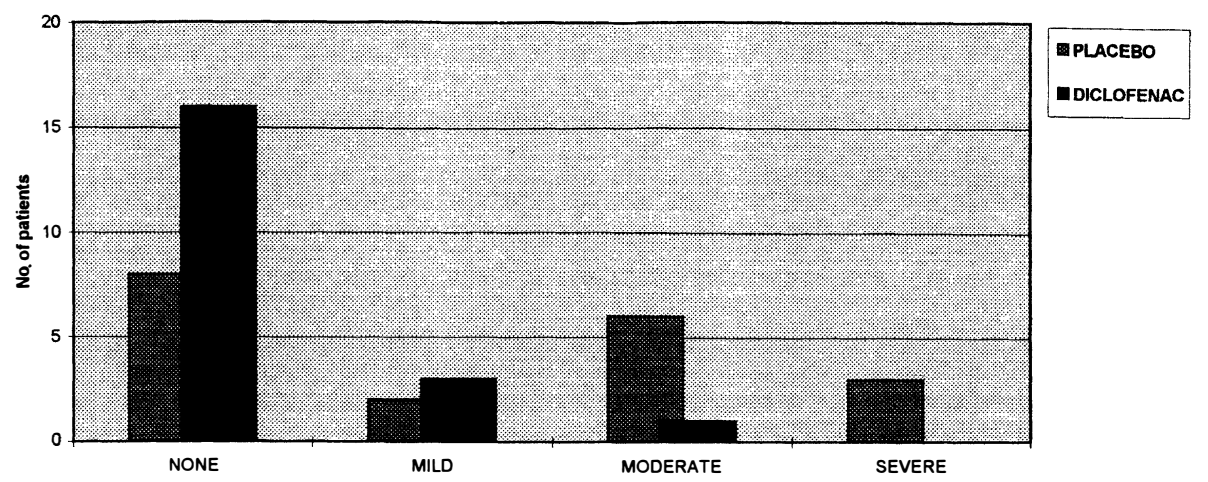

(c)

Fig. 3. Patient assessment of (a) foreign body sensation, (b) light sensitivity (photophobia) and (c) headache-like pain following traumatic corneal abrasion. Each graph represents the mean from 20 patients in each group during the first 48 hours.

of prostaglandins. Prostaglandin $\mathrm{E}_{2}$ is generally thought to be responsible for inflammation and pain.

Prostaglandin-synthesising capacity exists in the corneal epithelium and stroma and increases rapidly after injury. ${ }^{9}$ It is likely that the analgesic properties of diclofenac are produced through a decrease in the production of prostaglandins ${ }^{10}$ and may also be related to increases in beta-endorphin production. ${ }^{11}$ Corneal nerve conduction is also depressed by topical diclofenac. $^{12}$

Systematic assays of patient discomfort are widely used in the field of clinical pharmacology regarding pain and pain relief ${ }^{13,14}$ and it is accepted that the analogue scale provides a very sensitive 'betweentreatment' comparison when measuring ocular dis- comfort. ${ }^{15}$ In this study, the visual analogue scale and categorical scales both demonstrated that diclofenac is significantly more effective than placebo at reducing discomfort following traumatic corneal abrasions. Topical diclofenac also reduced the need for oral analgesics, as demonstrated by the statistical analysis of the categorical pain scale data and the degree of pain and light sensitivity.

The treatment regimen of topical diclofenac sodium $(0.1 \%)$ and antibiotic ointment 4 times daily as outlined in this article appears to provide a superior alternative to the traditional treatment of corneal abrasions.

Key words: Corneal abrasions, Diclofenac, Non-steroidal antiinflammatory drugs (NSAIDs). 


\section{REFERENCES}

1. Sher NA, Frantz JM, Talley A, et al. Topical diclofenac in the treatment of ocular pain after excimer photorefractive keratectomy. Refract Corneal Surg 1993; 9:425-36.

2. Dua HS, Forrester JV. The corneoscleral limbus in human corneal epithelial wound healing. Am J Ophthalmol 1990;110:646-56.

3. Yang Z, Zhao Z, Panjwani N. Gangliosides of migrating and nonmigrating corneal epithelium in organ and cell culture. Invest Ophthalmol Vis Sci 1996;37:501-10.

4. Ku EC, Lee W, Kothari HV, Scholer DW. Effect of diclofenac sodium on the arachiodonic acid cascade. Am J Med 1986;80(Suppl):18-23.

5. Hulbert MFG. Efficacy of eye pad in corneal healing after corneal foreign body removal. Lancet 1991;337:643.

6. Kantor TG. Current modalities in arthritic disease. Am J Med 1987;83(Suppl 4B):2-5.

7. Loya N, Bassage S, Vyas S, Del Cerro M, Park SB, Aquavella JV. Topical diclofenac following excimer laser: effects on corneal sensitivity and wound healing in rabbits. J Refract Corneal Surg 1994;10:423-7.
8. Tripathi BJ, Tripathi RC, Kolli P. Cytotoxicity of ophthalmic preservatives on human corneal epithelium. Lens Eye Toxicity Res 1992;9:361-75.

9 Bazan HEP. The synthesis and effects of eicosanoids in avascular ocular tissues. In: Bito LZ, Stjernschantz J, editors. The ocular effects of prostaglandins and other eicosanoids. New York: AR Liss, 1989:73-84.

10. Scholer DW, Ku EC, Boettcher I, Schweizer A. Pharmacology of diclofenac sodium. Am J Med 1986;80(Suppl):34-8.

11. Martini A, Bondiolotti GP, Sacerdote $\mathrm{P}$, et al. Diclofenac increases beta-endorphin plasma concentrations. J Int Med Res 1984;12:92-5.

12. Bauerman RW, McDonald MB, Varnell RJ, Thompson HW. Neurophysiological evaluation of corneal nerves in rabbits following excimer PRK [abstract]. Invest Ophthalmol Vis Sci 1993;34:704.

13. Huskisson EC. Measurement of pain. Lancet 1974;2:1127-31.

14. Aitken RCB. Measurement of feelings using analog scales. Proc R Soc Med 1969:62:989-92.

15. Scolville B, Krieglstein GK, Then E, Yokoyama S, Yokoyama T. Measuring drug-induced eye irritation: a simple assay. J Clin Pharmacol 1985;25:210-8. 